\title{
Aportes ricoeurianos para la transformación metapsicológica del psicoanálisis de D.W. Winnicott: fuerza, sentido y transicionalidad
}

\author{
Julieta Bareiro \\ Universidad de Buenos Aires
}

Resumen: El presente artículo se inscribe dentro de la tradición interdisciplinaria e intenta hacer un aporte en el campo de las relaciones entre psicoanálisis y fenomenología hermenéutica. Puntualmente, la contribución se centra en señalar la transformación que Winnicott realiza de la metapsicología freudiana. Para lograr este propósito, se asume como criterio de análisis de la obra de Winnicott las reflexiones fenomenológico-hermenéuticas de la lectura que P. Ricoeur hace de la metapsicología freudiana en Freud: una interpretación de la cultura (1985).

Palabras clave: Ricoeur; Winnicott; Freud; psicoanálisis; metapsicología

\begin{abstract}
Ricoeurian Contributions to the Metapsychological Transformation of D. W. Winnicott's Psychoanalysis: Strength, Meaning and Transitionality". This article is part of the interdisciplinary tradition and attempts to make a contribution in the field of the relations between psychoanalysis and hermeneutical phenomenology. Particularly, the contribution lies in pointing out the transformation Winnicott makes out of the Freudian metapsychology. To carry out this purpose, the phenomenological-hermeneutic reflections of the reading P. Ricoeur makes of Freudian metapsychology in Freud: an Interpretation of Culture (1985) will be assumed as an analysis criterion of Winnicott's work.
\end{abstract}

Keywords: Ricoeur; Winnicott; Freud; psychoanalysis; metapsychology 
La articulación entre psicoanálisis y fenomenología hermenéutica tiene un recorrido fecundo. A lo largo del siglo XX hubo numerosos abordajes del cruce entre ambos campos teóricos. En el ámbito del pensamiento francés, por ejemplo, comenzado por el psicoanálisis existencial de Sartre, continuando con la fenomenología del cuerpo de Merleau-Ponty, pasando por el enfoque epistemológico-hermenéutico de Paul Ricoeur, la incorporación de los problemas de psicoanálisis en la arqueología del saber de Foucault y llegando hasta la fenomenología material de Henry, se cuenta con una larga tradición de reflexión interdisciplinaria.

La obra de Winnicott se sitúa en este contexto de discusión y requiere de una reflexión metodológica particular a fin de poder ser abordada desde un punto de vista fenomenológico-hermenéutico. La razón de ello es que Winnicott nunca tuvo dentro de sus fines proponer un sistema de pensamiento cerrado, tal como advierten M. Davis y D. Wallbridge ${ }^{1}$ y Green ${ }^{2}$. Una de las razones de esta asistematicidad del marco teórico radica en el público al que Winnicott se dirige: padres, asistentes sociales, educadores, enfermeras, etcétera. Uno de los riesgos de esta política enunciativa es la banalización de sus conceptos. Para evitar este riesgo y, fundamentalmente, porque el pensamiento winnicottiano encierra una transformación profunda del psicoanálisis, es necesario analizar las consecuencias metapsicológicas y clínicas que solo están indicadas en su obra. Con el fin de lograrlo, se asume como criterio de análisis de la obra de Winnicott las reflexiones fenomenológico-hermenéuticas de la lectura que Ricoeur hace de la metapsicologia freudiana. Sin embargo, cabe aclarar que Ricoeur menciona a Winnicott en Caminos del reconocimiento ${ }^{3}$ al señalar los conceptos de Honneth en La lucha por el reconocimiento ${ }^{4}$ en relación con los lazos pre-jurídicos entre los individuos. En ese texto, Ricoeur destaca la importancia del espacio transicional como un ámbito no tensivo, distinto a la

Cf. Davis, M. y D. Wallbridge, Límite y espacio, Buenos Aires: Amorrortu, 1988.

Cf. Green, A., Jugar con Winnicott, Buenos Aires: Amorrortu, 2002.

Ricoeur, P., Caminos del reconocimiento, Madrid: FCE, 2006.

4 Cf. Honneth, A., La lucha por el reconocimiento: por una gramática moral de los conflictos sociales, Barcelona: Critica, 1997. 
realidad exterior e interior y donde se despliega la cultura como la matriz lúdica del adulto ${ }^{5}$. Esta mención, profundiza aun más los lazos entre ambos autores.

El recorrido de este trabajo es el siguiente: en la primera parte, se aborda la noción de transicionalidad de Winnicott y cómo este concepto reformula la metapsicología freudiana en relación con la pulsión. En la segunda parte, se analiza la lectura de Ricoeur sobre el discurso mixto del psicoanálisis freudiano y la tensión entre fuerza y sentido de la metapsicologia. En el final de esta parte, se vincula a los autores mencionados para establecer puentes en relación con las nociones de espacio, fuerza y sentido. Por último, a modo de cierre, se señala otros vasos comunicantes sobre el simbolo y la cultura

\section{Transicionalidad en el psicoanálisis de D. W. Winnicott}

El concepto fundamental de Winnicott de la transicionalidad es una noción que tiene implicancias metapsicológicas y clinicas. La metapsicología fue introducida por Freud en una carta a Fliess del año 1896. Dicha noción expresa el trabajo de justificación teórica de los conceptos fundamentales de la clínica del psicoanálisis. Por este motivo es que P.L. Assoun 6 afirma que la metapsicología es el intento de validación del psicoanálisis como saber, es decir, expresa la tarea epistemológica que hace del psicoanálisis un saber específico y singular. De ahí que toda tarea de fundamentación en el psicoanálisis tenga el carácter de una metapsicología. La metapsicología es un modo de concepción, según el cual todo proceso psíquico es apreciado según las tres coordenadas (tópica, dinámica y económica). Según Assoun, estas coordenadas se basan en el modelo de la física al que Freud adhiere el psicoanálisis como "ciencia de la naturaleza" [Naturwissenschaft] que piensa a los cuerpos en términos de proyección espacial, de despliegue de fuerzas y de producción de cantidades. Las metáforas fisicoquímicas dan prueba de esta referencia.

\footnotetext{
5 Ricoeur en un pie de página cita a Honneth citando a Winnicott: "Suponemos que la aceptación de la realidad es una tarea nunca concluida enteramente, y que ningún ser humano llega a liberarse de la tensión suscitada por la carga de tener que poner en relación la realidad interior y la realidad exterior; suponemos también que esta tensión puede mitigarse por la existencia de un dominio intermediario que no es cuestionado (arte, religión, etc.). Este dominio intermediario se desarrolla directamente a partir del espacio lúdico en el que evoluciona el niño 'perdido' en su juego' (D. W. Winnicott, Reality and Playing, citado por A. Honneth en La Lutte pour la reconnaieeance, cit., p. 126)" (Caminos del reconocimiento, p. 241).

${ }^{6}$ Cf. Assoun, P., Introducción a la metapsicología freudiana, Buenos Aires: Paidós, 1994; La metapsicología, Buenos Aires: Siglo XXI, 2002.
} 
El estatuto de este saber metapsicológico fue muy discutido después de la muerte de Freud. Las distintas escuelas postfreudianas asumieron posiciones divergentes acerca de la legitimidad de esta tarea: basta señalar los aportes de Lacan $^{7}$ y Klein ${ }^{8}$ para darse cuenta de la importancia del problema metapsicológico en el psicoanálisis. La obra de D.W. Winnicott se sitúa en ese contexto de discusión. En sus escritos se pueden encontrar distintas expresiones en la que manifiesta su desinterés ante los problemas de fundamentación. Aparentemente renuncia a la tarea de formación teórica de las nociones fundamentales de su pensamiento para presentarse "tan solo" como un clínico preocupado en el sufrimiento psíquico de sus pacientes. Esta indiferencia hacia la metapsicologia puede encontrarse, por ejemplo, en las afirmaciones que aparecen en The Maturational Processes and the Facilitating Environment 9 . No obstante, desde hace unos años distintos investigadores comenzaron a ver en los textos del psicoanalista inglés una auténtica reflexión metapsicológica que compite con la freudiana (tales como Loparic ${ }^{10}$ y Green ${ }^{11}$, entre otros).

El concepto metapsicológico fundamental de Winnicott es el fenómeno transicional (transitional phenomena), noción que aparece por primera vez en el artículo "Objects and Transitional Phenomena" (1951) ${ }^{12}$. En 1971, en su libro más conocido, Reality and Playing, retoma y reelabora esta noción a raíz de que, según afirma, la transicionalidad no fue debidamente comprendida, quedando ligada a la mera descripción del oso de peluche o la manta acogedora. Las críticas que Winnicott hace a la recepción de su concepto son varias: a) que se ha dejado de lado la importancia de la transicionalidad como tercer área del individuo, donde habita el verdadero sí mismo; b) que el fenómeno transicional permite comprender la cultura como experiencia del propio-ser (true self); c) que es un fenómeno universal, y d) que es una meta clínica.

Bajo este concepto, Winnicott procuró en sus postulados teóricos ubicar al sujeto en relación con su propia potencialidad. En este sentido, se diferencia de otros autores como Freud y Klein. Para estos, lo pulsional era el factor decisivo constitutivo del psiquismo. Para Winnicott, en cambio, el fenómeno

7 Cf. Lacan, J., Escritos I, Buenos Aires: Siglo XXI, 2006.

8 Cf. Klein, M., Obras completas, v. I y v. II, Buenos Aires: Paidós, 2008.

9 Winnicott, D.W, The Maturational Processes and the Facilitating Environment, Nueva York: International Universities Press, 1965, pp. 96-97.

${ }^{10} C f$. Loparic, S., "Além do inconsciente: sobre a desconstrução heideggeriana da psicanálise", en: www.naturezahumana.br., 2002.

${ }^{11}$ Cf. Green, A., Jugar con Winnicott, Buenos Aires: Amorrortu, 2002.

12 Winnicott, D.W., Collected Papers; through Paediatrics to Psychoanalysis, Nueva York: International Universities Press, 1958. 
transicionaly su concomitante acto creativo expresan la singularidad misma de la subjetividad. Por fenómeno transicional se comprende aquella experiencia que no es ni interna (subjetiva) ni externa (percibida objetivamente) aunque involucre a ambas. Es una modalidad de funcionamiento psíquico que constituye los fenómenos, el espacio y los objetos transicionales. Implica la constitución del mundo y el modo de habitar del sujeto en ese proceso. Son fenómenos de características ilusorias que, partiendo de una indistinción entre lo subjetivo y lo que es exterior al sujeto, devienen en ámbitos y procesamientos distinguibles y relacionables. En sus comienzos, comprende la creación de un objeto preexistente por parte del bebé. El bebé crea un mundo que ya ha sido creado, pero que está a la espera de significación. Esta experiencia, que denomina paradójica, lleva al infante a la posibilidad de simbolización. Para Winnicott la capacidad de crear es la manifestación de sentirse vivo, verdadero y real, en la medida que la posibilidad de la experiencia del fenómeno transicional es la experiencia de la propia existencia.

El sentido de lo originario del fenómeno transicional se ubica en los primeros actos del bebé que pasa del cuerpo propio al objeto ajeno experimentado como la primera posesión no-yo. El oso de peluche o la manta acogedora son ejemplos de estas primeras posesiones que involucran a la capacidad creadora que les otorga significado. El entorno mediato o inmediato del bebé se ofrece a su intención significativa primaria. Esta creatividad, propia de los fenómenos transicionales, se mantiene constante y abarca desde las etapas tempranas hasta la adultez. Las experiencias culturales, el arte, la ciencia y la religión son destacados en relación con lo transicional.

Este ámbito de significación se denomina espacio transicional (transitional space) en el cual contribuyen la realidad exterior e interior. La realidad interior corresponde al psiquismo freudiano, mientras que la exterior corresponde a la realidad efectiva. El espacio transicional es virtual, se abre entre la subjetividad del infante y el reconocimiento del mundo exterior. Esta tercera zona de la experiencia, como la denomina Winnicott, se diferencia de las otras dos en que no es objeto de desafio alguno. Ni los avatares de la realidad se le imponen, ni lo pulsional la domina. El espacio transicional es el ámbito del sí mismo, que se ubica como un delicado espacio de juego entre los otros dos espacios: ni plenamente subjetivo, ni enteramente objetivo. Por el contrario, más que un encauzar a las fuerzas pulsionales o a las demandas externas, implica un relajamiento de ellas y, debido a ello, un lugar de descanso que involucra, paradójicamente, a ambas. Presupone a un self que, en tanto verdadero, es 
genuinamente sí mismo y que, en virtud de ello, la capacidad creadora es el reflejo de esta realidad.

De esta forma, el fenómeno, espacio y objeto transicional se establecen como aquellas categorias por medio de las cuales Winnicott interpreta lo sano y lo enfermo, la verdad y la falsedad del sí mismo, la libertad y el acatamiento y, en general, el vínculo con el mundo. La transicionalidad se presenta como un fenómeno que se anuncia en la clínica psicoanalítica, pero por sus definiciones comprende el carácter de lo originario de la subjetividad. Winnicott reconoció que no se restringe al espacio cerrado de la praxis clínica, sino que se extiende a otros saberes y ámbitos hasta caracterizar por completo a la cultura misma ${ }^{13}$.

Esta perspectiva produce un giro conceptual respecto de lo pulsional como lo más originario. La inversión de la metáfora del caballo que utiliza Winnicott para distanciarse de la sobredeterminación pulsional de Freud resulta ilustrativa. En el capitulo 7 de Reality and Playing ("The Location of Cultural Experience") se pregunta qué se entiende por vida y cuándo un sujeto comienza a ser. Desecha la idea de que lo instintivo domine y advierte la peligrosidad que este tendría sobre los fenómenos transicionales. Para Winnicott, en las etapas tempranas, el sujeto se encuentra en un estado de pura necesidad de ser cuidado y de dependencia al ambiente. La problemática de la pulsión recién surge a partir de la integración del ego como cambio cualitativo. Incluso plantea que de no mediar este, la cuestión pulsional "puede ser tan externa como puedan serlo los truenos o los golpes. El ego de la criatura está haciendo un acopio de fuerzas y, por consiguiente, acercándose a un estado en que las exigencias del id serán percibidas como parte del ser, en lugar de serlo como factores ambientales" ${ }^{14}$. Para el autor, primero están las necesidades de ser y existir y, luego, las satisfacciones de la pulsión. Es decir, no es la satisfacción instintiva el factor primordial para que un bebé empiece a ser. A esta energía Winnicott la denomina agresividad. Esta va desde la musculatura hasta caminos cada vez más sofisticados, como la creación de objetos y la significación paulatina

$288{ }^{13}$ Winnicott, D.W., Reality and Playing, Nueva York: Basic Books, 1971, p. 22.

${ }^{14}$ Winnicott, D.W., Psychoanalitics Explorations I and II, Nueva York: International Universities Press, 1972, p. 171. Sin embargo, existe un problema terminológico: la traducción de trieb por instinct o drive. Levin de Said establece que Winnicott "denomina instinto (instinct) a las poderosas mociones biológicas (drive) que van y vienen en la vida del bebé y del niño y que demandan una acción. No obstante, no considera necesaria una clasificación del instinto. Sí le importa "la elaboración imaginativa del funcionamiento corporal", concepto intrinsecamente ligado con el de "ausencia". Dicha idea posee imágenes cercanas al concepto de trabajo de la pulsión de Freud como exigencia de trabajo". Esta cita la encontramos en: Levin de Said, A., El sostén del ser: las contribuciones de D. W. Winnicott, Buenos Aires: Paidós, 2006, p. 90. 
del mundo circundante. Sin embargo, esta fuerza agresivamente amorosa solo lo es como potencialidad. No habria antítesis entre vida o muerte, ni mezcla ni desmezcla pulsional. Es una potencia que incluye tanto a una como a la otra, pero orientada hacia una creatividad que toma impulso de esa misma agresividad. Aquí entra la cuestión del ambiente como marco y sostén. Un ambiente lo suficientemente bueno da lugar a la experiencia del impetu vital. Los objetos, si todo va bien, son creados y potencialmente destruidos por esta fuerza. Esta destructividad potencial, creativa y creadora, posibilitadora de la alteridad, no es producto de la pulsión. Solo en la medida en que la pulsión logra integrarse al ego, aquella adquiere el valor energético y de empuje conceptualizado tradicionalmente por el canon psicoanalítico, lo que implica una subversión de la lógica freudiana. Así, toma valor la cuestión del self como una unidad integrada, que en varias ocasiones Winnicott acerca a la noción de Yo que crece hasta lo que denomina núcleo del propio ser como fuerza motora. Debe entenderse que toda experiencia -incluida la de la fuerza pulsional- no existe sin un yo que posibilite esa misma experiencia.

Otros autores, como Levin de Said ${ }^{15}$, entienden que la cuestión de la pulsión freudiana en Winnicott aparece vinculada a la fuerza vital, que encuentra comparable a las mociones eróticas y agresivas que recorren diversos caminos. En este sentido, plantea que el vocabulario winnicottiano utiliza más la idea de impulsos instintivos que el término pulsión. Por su parte, Davis y Wallbridge deducen que "como el Ello de Freud, el propio ser central de Winnicott es la fuente de energía y espontaneidad. Pero Winnicott no otorga la misma primacía que Freud a las pulsiones sexuales y agresivas: las considera más bien tributarias de los procesos de maduración... Winnicott entendía que, antes que pudiera hacer uso alguno de esos instintos, tenia que estar como una persona vivenciante, por más rudimentaria que fuera" ${ }^{16}$.

Es claro que en la recepción de la obra habría acuerdo en cuanto a lo no originario de la pulsión. En su lugar se ubica la cuestión del núcleo del ser como fuerza vital y al desarrollo del yo como mediador de las experiencias pulsionales. $\mathrm{El}$ aporte de Winnicott sobre la tradición freudiana es la del cambio respecto de la segunda tópica, donde el organismo tiende a lo vital. Cualquier vinculación con lo tanático remite a fallas extremas del entorno en tanto cuidado y sostén, profundizando el abismo del desamparo primordial hasta la extinción

15 Levin de Said, A., El sostén del ser: las contribuciones de D. W. Winnicott, p. 91.
16 Davis, M. y D. Wallbridge, Limite y espacio, p. 45.

ARETÉ Revista de Filosofía, vol. XXXI, N² 2, 2019 / ISSN 1016-913X 
misma. En su lugar ubica a la agresividad primaria compuesta de elementos amorosos y destructivos. Esta destructividad no pretende hacer daño y, de hacerlo, ocurre "solo por azar". Así habría un primer dualismo que refiere de esta manera: "...uno de los fenómenos integradores del desarrollo de la fusión de lo que aquí me permitiré a mí mismo llamar instintos de vida y de muerte (de amor y de discordia en Empédocles). El eje de mi argumentación es que la primera moción es, en sí misma, una sola, es algo que yo llamo destrucción, pero también podría haber llamado moción combinada de amor y discordia. Esta unidad es primaria. Es lo que sale a relucir en el bebé, por los procesos naturales de maduración"17.

Si en la teoría naturalista de Freud lo pulsional resulta dominante y, sobre todo, la marca de la pulsión de muerte, en Winnicott la agresividad pulsa hacia una creatividad cuyo propósito se orienta hacia lo espontáneo. Si Freud en la imagen del caballo y del jinete invierte las figuras para señalar lo dominante de lo pulsional, Winnicott vuelve a modificarlo, e indica que es la capacidad de crear lo que hace que un sujeto se sienta "vivo, verdadero y real". Es alli donde lo pulsional tiene factor de fuerza, más que de dominio; de lo contrario, puede ser "tan externo como un rayo". Así, la capacidad creadora refleja la agencia del propio-ser y no la mera compulsión. Desde esta posibilidad, la relación entre el sujeto y el mundo lo constituyen los fenómenos transicionales.

Ahora bien, aunque Winnicott no mostró pretensiones más allá de dar cuenta de su clinica, con su concepto de transicionalidad y el cuestionamiento de lo pulsional como dominante y con la idea de una tercera zona de la experiencia, plantea un corrimiento respecto de la metapsicología freudiana. Para señalar este "giro" -por decirlo así- se tomará como clave de análisis la lectura del psicoanálisis de P. Ricoeur.

\section{El psicoanálisis desde la lectura de P. Ricoeur en "Freud: una interpreta- ción de la cultura"}

Ricoeur divide su lectura de Freud en una Analítica y una Dialéctica. 290 Llama Analitica a la primera lectura de Freud porque es una lectura en inmanencia, es decir, no confrontada con otras interpretaciones. La Dialéctica es la incorporación de las otras interpretaciones, ajenas y exteriores al psicoanálisis. Ricoeur hace una aclaración hegeliana de la Dialéctica: "Solo para la segunda lectura, la oposición exterior y totalmente mecánica de los puntos de vista podrá

${ }^{17}$ Winnicott, D.W., Psychoanalitics Explorations I and II, p. 292. 
investirse en oposición interna y cada punto de vista resultará en alguna forma su contrario y contendrá dentro de sí la razón del punto de vista inverso"18.

Por su parte, la exposición analítica tiene un carácter abstracto. Ello da cuenta del modo en que interpreta el estatuto epistémico del psicoanálisis. Para Ricoeur se trata de una ciencia humana y no de una ciencia natural, en el sentido de que la teoría fundamenta los hechos mismos. Esto es, que se determinan por su metapsicología. Aquí, el propósito es dar cuenta de su epistemología, es decir, "de una investigación de los enunciados del psicoanálisis y de su situación del discurso"19.

Para Ricoeur, el problema central de la epistemología freudiana reside en su condición de discurso mixto entre fuerza y sentido. Esta tesis del carácter mixto lleva consigo una dificultad: el género del discurso energético posee un carácter antifenomenológico cuya consecuencia es que daría cuenta de una explicación naturalista y excluiría una explicación hermenéutica. A partir de esta dificultad inherente a la tópica y la económica, Ricoeur formula el problema epistemológico: "Me parece que todo el problema de la epistemología freudiana se concentra en este único problema ¿cómo es posible que la explicación económica para una interpretación referida a significaciones $\mathrm{y}$, a la inversa, que la interpretación sea un momento de la explicación económica? Lo fácil es lanzarse a una alternativa: o una explicación de índole energética o una comprensión de indole fenomenológica. Ahora bien, es preciso admitir que el freudismo existe a costa de rechazar esa alternativa"20.

El concepto que, a su juicio, logra superar la distancia de los dos géneros del discurso es el deseo que permite el pasaje de la fuerza al lenguaje como también la de integrar aquella. Ricoeur llamará "semántica del deseo" al propósito de la teoría freudiana de situar el trabajo de la interpretación en la región del deseo: "Freud invita a buscar en el sueño mismo la articulación del deseo y del lenguaje; y esto de múltiples maneras: primero, no es el sueño soñado lo que puede ser interpretado, sino el texto del relato del sueño; es a este texto al que el análisis quiere sustituir por otro texto que sería como la palabra primitiva del deseo; de modo que el análisis se mueve de un sentido a otro sentido; de ningún modo es el deseo como tal lo que se haya situado en el centro del análisis, sino su lenguaje"21.

\footnotetext{
${ }^{18}$ Ricoeur, P., Freud: una interpretación de la cultura, p. 56.

19 Ibid., p. 133.

20 Ibid., p. 61.

21 Ibid., p. 9.
} 
Lo que importa plantear desde el principio es que esta dinámica —o esta energética, y aun esta hidráulica - del deseo y la represión no se enuncia sino en una semántica: las vicisitudes de las pulsiones, para retomar un término de Freud, no pueden alcanzarse más que en las vicisitudes del sentido. En ese sentido, es posible hablar aquí, con Ricoeur, de una "semántica del deseo". Para Corona, este término señala la concepción de la arqueología de Freud, según la cual todo el orbe de las representaciones de la conciencia, desde lo infantil hasta las sublimes representaciones de la religión, nace del deseo y para el deseo; deseo que sigue siendo siempre, enmascarado a través de todas las esferas no vitales de la conciencia, el deseo infantil, el deseo de las pulsiones de autoconservación y sexuales ${ }^{22}$. Así, el pensamiento acontece en el límite con lo que lo excede. Puede advertir ese límite, pero no por ello puede sobrepasarlo y apoderarse de lo que se halla más allá para lograr con ello una homogeneidad de "naturaleza". Sea cual fuere la pulsión que presida la constelación pulsional constitutiva del sujeto dado, prima lo afectivo.

La condición de texto del sueño en La interpretación de los sueños ${ }^{23}$ conduce a que sea considerado como totalidad significativa para la metapsicología al definirlo como discurso mixto y paradigma para comprender el síntoma: "si el sueño tiende por su carácter de relato al discurso, la voluntad de poder, la libido o como quiera decirse. De este modo, el sueño como expresión del deseo, se encuentra entre el sentido y la fuerza"24. La estructura mixta del sueño halla su correlato en la noción de interpretación (Deutung). Es muy interesante notar la lectura que hace sobre este término al conferirle un sentido tópico como desplazamiento de lugar que pone de relieve, aunque sin decirlo, la condición deíctica de la interpretación freudiana. Interpretar significa dirigir la vista hacia otro lugar: "la interpretación es un movimiento de lo manifiesto a lo latente; interpretar es trasladar el origen del sentido hacia otro lugar"25 (ídem). El afirmar que el sueño es la realización de un deseo reprimido supone conjugar dos conceptos que pertenecen a órdenes diferentes: la realización pertenece al discurso del sentido y la represión al de la fuerza.

Ricoeur señala la condición paradojal del psicoanálisis producto de su propia base conceptual. El factor energético pulsional posee la particularidad de que no aparece de forma pura sino mediada por la dimensión significativa

\footnotetext{
${ }^{22}$ Corona, N., "La lectura ricoeuriana de Freud sobre la cuestión del sujeto", en: Revista Latinoamericana de Filosofia, v. XXXII, 2 (2006), p. 30.

${ }^{23}$ Cf. Freud, S., "La interpretación de los sueños", Buenos Aires: Amorrortu: 1996.

${ }^{24}$ Ricoeur, P., Freud: una interpretación de la cultura, pp. 81-82.

${ }^{25}$ Ibid.
} 
del Repräsentanz (presentación de la pulsión). Esta noción deja de lado la condición biológica de la pulsión y deja en su lugar el "ser psicológico de las representaciones psíquicas de la pulsión". Ello plantea una serie de problemas: ¿Cómo se vinculan las fuerzas de la pulsión con sus representantes? ¿Cuál es la relación entre ambas con el deseo? Para Ricoeur la respuesta se halla en el estatuto mixto del discurso psicoanalítico donde se enlazan ambas instancias opuestas: fuerza y sentido. La parte pulsional como energética dará cuenta de la condición dinámica del aparato psíquico, mientas que la hermenéutica formula el polo significativo, discursivo, del mismo. Así, Ricoeur encuentra en la obra freudiana un desplazamiento de esta dualidad que va desde una versión de tipo naturalista del aparto psíquico a una orientada a la condición significativa. Ello puede encontrarse, por ejemplo, en las referencias de "Proyecto de una psicología para neurólogos" (1895), donde las nociones de psiquismo, constancia y fuerza reflejan el modelo de artefacto y cantidades. Tres años después, en "La sexualidad en la etiología de la neurosis" (1898) añade la relación entre analista-paciente mediatizada por el discurso entre ambos. E1 gran cambio epistémico surge en "La interpretación de los sueños" ${ }^{26}$, donde el modelo de aparato cambia de una configuración física a una analógica ${ }^{27}$. Sin embargo, estos cambios nunca anulan la condición energética del psiquismo. Baste recordar las definiciones pulsionales en "Lo inconsciente" (1915) o los "vasallajes del yo" en el capítulo V del "Yo y el Ello" (1923) La particularidad es que pulsión muta de fuerza desnuda a concepto límite entre lo físico y lo psíquico. Para Ricoeur, esto indica que el psicoanálisis se ocupa de la representación de la pulsión [Repräsentanz] y no de la pulsión misma.

Así entonces, ¿de qué manera comprender la relación entre fuerza y sentido? ¿Cómo repensar la condición mixta del psicoanálisis? Una vía posible es a través de la semiótica de I. Lotman. En otro trabajo ${ }^{28}$ se abordó el problema del discurso científico como desmundanización en la filosofia de Heidegger y el psicoanálisis de Freud. En ese artículo, la semiótica de I. Lotman permitía dar cuenta de la transición entre sentido y sinsentido a través de lo que el semiólogo ruso denomina semiósfera o biosfera semiótica, esto es, el universo de signos donde la humanidad vive e interactúa. Dichos signos son representaciones que

${ }^{26}$ Cf. Freud, S., La interpretación de los sueños, Buenos Aires: Amorrortu: 1996.

${ }^{27}$ Ricoeur, P., Freud: una interpretación de la cultura, p. 94.

${ }^{28}$ Bareiro, J. y A. Bertorello, "Lógica de la diferencia y lógica de la alteridad. Sentido y sinsentido en Heidegger y Winnicott", en: Anuario de Investigaciones de la Facultad de Psicología, v. XVII (2010), pp. 275-282. 
establecen un espacio reticulado respecto de lo que lo rodea, que se denomina extrasemiótico. En la separación entre lo semiótico y lo extrasemiótico emerge una frontera que actúa como filtro entre ambos espacios y que le otorga sentido a lo extrasemiótico dentro de alguno de los sistemas semióticos. Con la noción de traductor filtro se refiere a un espacio de frontera irregular y heterogénea cuya función es la de habilitar la interacción de dos registros diferentes dando como resultado la producción de sentido. Con este modelo, es posible comprender que para Ricoeur es el deseo el que realiza esa labor de traductor filtro entre los elementos pulsionales y sus representaciones ${ }^{29}$.

Desde esta perspectiva, lo singular del discurso psicoanalitico es la pretensión de que en el mismo plano del sentido se da envergadura a la condición cósica del psiquismo. Esto es, que es en ese mismo ámbito donde se da cuenta del sinsentido, del polo energético que se resiste a la significación. Winnicott introduce una lectura novedosa al darle mayor relevancia al plano del sentido que al de la fuerza. En efecto, la noción de transicionalidad se define por ser un espacio no tensivo, de dinámica diferente a la del principio de la realidad como a la de la fuerza pulsional. La denominación de Winnicott del espacio transicional como "tercera área de la vida" renueva el rasgo topológico del psiquismo a la vez deja en un segundo plano las otras dos condiciones tradicionales de la metapsicología freudiana. Por un lado, el polo energético, ya que lo pulsional se subsume a la condición creativa del sí mismo y, por el otro, el económico, que diluye lo tensivo y deja en su lugar a este espacio como una red espacial significativa. Así señalado, Winnicott propone, sin buscarlo, una renovación de la metapsicología freudiana. Si bien en sus escritos "tan solo" pareciera ocuparse de la clínica, sus postulados sobre el carácter de la transicionalidad y sus conceptos lindantes (sí mismo, creatividad, objeto transicional, experiencia cultural, tercera área de la vida, etc.) provocan una reformulación sobre lo interno, lo externo y lo pulsional a la vez que reformula el carácter naturalista del psiquismo. Éste ya no se enfrenta con las fuerzas desnudas de la pulsión, sino que su aparición se concibe como la fuerza creadora vital que hace a la significación del mundo. Esta noción no está desprovista de historicidad, en la medida que la trasmisión cultural conlleva la matriz histórica en la que existe.

Winnicott plantea que la vida humana, a diferencia de la vida biológica, transcurre en el plano de los significados culturales y que, por ello, la modalidad específica de la vida es la potencialidad. Es en el ámbito cultural donde se

${ }^{29}$ Ricoeur, P., Freud: una interpretación de la cultura, p. 380. 
despliega la vida humana. Esto no refiere a una relación objeto/sujeto, sino a la creación del mundo en la experiencia cultural. Su manifestación más clara es la capacidad de constituir un espacio potencial. Aquí aparece una gran diferencia respecto de la cultura en Freud y en Winnicott. Para el primero, la cultura es "el combate de la especie humana por la vida" 30 y debido a ello, posee un carácter conflictivo y coactivo donde se despliegan la agresividad, la hostilidad y la crueldad. Para Winnicott, en cambio, la cultura es un espacio que no tiene necesariamente un carácter conflictivo, sino más bien integrador. Aquí hay una perspectiva similar a la propuesta de Ricoeur sobre la obra de arte en el discurso psicoanalítico. Para el filósofo francés, la obra de arte implica semántica, creación y juego, y no meramente la expresión de un síntoma o malestar. Para Winnicott, la creatividad se extiende desde el objeto transicional de la infancia hacia las experiencias culturales tales como el arte, la religión y la ciencia. Esto se debe a que es el propio-ser auténtico el que vivencia y recrea ambos, tal como lo señala en Reality and Playing ${ }^{31}$.

De esta manera, es la cultura el campo originario del existir donde se vinculan lo propio y lo ajeno para transformarlo en la marca de lo auténtico. Winnicott plantea que la vida humana, a diferencia de la vida biológica, transcurre en el plano de los significados culturales y que por ello la modalidad específica de la vida es la potencialidad, esto es, crear un espacio de despliegue de posibilidades. Aquí aparecen el jugar y la creatividad como la capacidad de introducir en el mundo nuevos comienzos. Vale decir, que el jugar es el puente entre la simbolización y el fenómeno transicional.

Recibido: 01/06/2018

Aceptado: 02/04/2019

\section{Bibliografia}

Assoun, P., Introducción a la metapsicología freudiana, Buenos Aires: Paidós, 1994.

Assoun, P., La metapsicología, Buenos Aires: Siglo XXI, 2002.

Bareiro, J. y Bertorello, A., "Lógica de la diferencia y lógica de la alteridad. Sentido y sinsentido en Heidegger y Winnicott", en: Anuario de Investigaciones de la Facultad de Psicología, v. XVII (2010), pp. 275-282.

Corona, N., "La lectura ricoeuriana de Freud sobre la cuestión del sujeto", en: Revista Latinoamericana de Filosofia, v. XXXII, 2 (2006).

30 Freud, S., "El malestar en la cultura", Buenos Aires, Amorrortu: 1996, p. 70.

31 Winnicott, D.W., Reality and Playing, Nueva York: Basic Books, 1971. 
Davis, M. y Wallbridge, D., Limite y espacio, Buenos Aires: Amorrortu, 1988.

Eco, U., Decir casi lo mismo. Experiencias de traducción, Barcelona: Lumen, 2008.

Freud, S., "La interpretación de los sueños", en: Obras completas, v. IV Buenos Aires: Amorrortu, 1996.

Freud, S., "Pulsión y destinos de pulsión", en: Obras completas, v. XIV, 1996.

Freud, S., "Mas allá del principio de placer", en: Obras completas, v. XVIII, Buenos Aires: Amorrortu, 1996.

Freud, S., "El malestar en la cultura", en: Obras completas, v. XXI, Buenos Aires: Amorrortu, 1996.

Green, A., Jugar con Winnicott, Buenos Aires: Amorrortu, 2002.

Klein, M., Obras completas, tomo I y II, Buenos Aires: Paidós, 2008.

Lacan, J., Escritos I, Buenos Aires: Siglo XXI, 2006.

Lapanche, J. y Pontalis, J-B., Diccionario de Psicoanálisis, Buenos Aires: Amorrortu, 1996.

Levin de Said, A., El sostén del ser: las contribuciones de D. W. Winnicott, Buenos Aires: Paidós, 2006.

Lotman, I., La semiósfera I, Valencia: Cátedra, 1996.

Loparic, S., "Além do inconsciente: sobre a desconstrução heideggeriana da psicanálise", en: www.naturezahumana.br., 2002.

Honneth, A., La lucha por el reconocimiento: por una gramática moral de los conflictos sociales, Barcelona: Crítica, 1997.

Ricoeur, P., Freud: una interpretación de la cultura, Madrid: Siglo XXI, 1985.

Ricoeur, P., Caminos del reconocimiento, Madrid: FCE: 2006.

Winnicott, D.W., Collected papers; through Paediatrics to Psychoanalysis, Nueva York: International Universities Press, 1958.

Winnicott, D.W., The Maturational Processes and the Facilitating Environment, Nueva York International Universities Press, 1965.

Winnicott, D.W., Reality and Playing, Nueva York: Basic Books, 1971.

Winnicott, D.W., Psychoanalitics Explorations I and II, Nueva York: International Universities Press, 1972. 